\title{
Determinação das Substâncias Reativas ao Ácido Tiobarbitúrico como Indicador da Peroxidação Lipídica em Ratos Tratados com Sevoflurano *
}

\author{
Thiobarbituric Acid Reactive Substances as an Index of Lipid \\ Peroxidation in Sevoflurane-Treated Rats
}

Francisco José Lucena Bezerra ${ }^{1}$; Adriana Augusto Rezende ${ }^{2}$; Sara Jane Rodrigues ${ }^{3}$; Maria das Graças Almeida ${ }^{2}$

\begin{abstract}
RESUMO
Bezerra FJL, Rezende AA, Rodrigues SJ, Almeida MG - Determinação das Substâncias Reativas ao Ácido Tiobarbitúrico como Indicador da Peroxidação Lipídica em Ratos Tratados com Sevoflurano
\end{abstract}

JUSTIFICATIVA E OBJETIVOS: O sevoflurano é um éter fluorado de baixa solubilidade sangüínea e sua biotransformação ocorre por meio do sistema enzimático hepático oxidativo que envolve o citocromo P450 2E1. A peroxidação lipídica ocorre durante o processo de biotransformação dos éteres sob ação do citocromo P450, um dos possíveis mecanismos de toxicidade hepática e renal promovida por esses compostos. O objetivo deste estudo foi determinar os níveis de substâncias reativas ao ácido tiobarbitúrico (SRAT), como indicador da peroxidação lipídica, em ratos que receberam sevoflurano, previamente tratados ou não com isoniazida, indutor enzimático do citocromo P450 2E1. MÉTODO: Foram utilizados 42 animais, distribuídos aleatoriamente em 4 grupos que receberam respectivamente: G1 - oxigênio a $100 \% 1 \mathrm{l} . \mathrm{min}^{-1} / 60$ minutos por 5 dias consecutivos; G2 - sevoflurano a $4 \%$ em oxigênio a $100 \%, 1$ I. $\mathrm{min}^{-1} / 60$ minutos por 5 dias consecutivos; $\mathrm{G} 3$ - isoniazida (50 $\mathrm{mg} \cdot \mathrm{kg}^{-1}$.dia) por via intraperitoneal durante 4 dias consecutivos, em seguida foi tratado como o G1, no G4 isoniazida $50 \mathrm{mg} \cdot \mathrm{kg}^{-1}$. dia por via intraperitoneal durante 4 dias consecutivos, sendo tratado, posteriormente, como o G2. Após 12 horas do último tratamento, sacrificaram-se os animais e foi coletado o plasma para a análise das SRAT, sendo removido o lobo esquerdo do fígado e os rins para exame histológico.

RESULTADOS: Os resultados mostraram aumento nas taxas de SRAT no G3 e G4, com elevação discreta em G2. O estudo histológico revelou necrose focal no fígado de ratos pré-tratados com isoniazida (G3).

\footnotetext{
* Recebido do (Received from) Departamento de Análises Clínicas e Toxicológicas do Centro de Ciências da Saúde da Universidade Federal do Rio Grande do Norte, Natal, RN

1. Anestesiologista do Hospital Universitário Onofre Lopes da UFRN

2. Professor Doutor do Departamento de Análises Clínicas e Toxicológicas do Centro de Ciências da Saúde da UFRN

3. Professor Mestre do Departamento de Anatomia Patológica do Centro de Ciências da Saúde da UFRN
}

Apresentado (Submitted) em 09 de setembro de 2003 Aceito (Accepted) para publicação em 05 de março de 2004

Endereço para correspondência (Correspondence to) Dr. Francisco José Lucena Bezerra

Rua Almirante Tamandaré, 146/202 -Lagoa Nova

59054-560 Natal, RN

E-mail: fjlb70@yahoo.com

(C) Sociedade Brasileira de Anestesiologia, 2004
CONCLUSÕES: O sevoflurano promoveu peroxidação lipídica apenas quando associado à isoniazida.

Unitermos: ANESTÉSICOS, Volátil: sevoflurano; ANIMAL: rato; DROGAS: isoniazida; METABOLISMO: peroxidação lipídica

\section{SUMMARY}

Bezerra FJL, Rezende AA, Rodrigues SJ, Almeida MG - Thiobarbituric Acid Reactive Substances as an Index of Lipid Peroxidation in Sevoflurane-Treated Rats

BACKGROUND AND OBJECTIVES: Sevoflurane is a fluorinated ether with low blood solubility and biotransformed by an oxidative enzymatic liver system involving cytochrome P450 $2 E 1$. Lipid peroxidation occurs during ethers biotransformation process under action of cytochrome P450, a possible mechanism for liver and kidney toxicity promoted by such compounds. This study aimed at determining the levels of substances reactive to thiobarbituric acid (TBARS), as an index for lipid peroxidation in sevoflurane-treated rats, previously treated or not with isoniazid, enzymatic inducer of cytochrome P450 2E1.

METHODS: Forty two male Wistar rats were randomly distributed in 4 groups receiving respectively: G1 - 1 L. min $^{-1} / 60$ minutes of $100 \%$ oxygen for 5 consecutive days; G2 - 4\% sevoflurane in $1 \mathrm{~L}$. $\mathrm{min}^{-1} / 60$ minutes of $100 \%$ oxygen for $5 \mathrm{con}$ secutive days; $\mathrm{G} 3$ - intraperitoneal isoniazid (50 mg. $\mathrm{kg}^{-1} / \mathrm{day}$ ) for 4 consecutive days and then treated as G1; G4 intraperitoneal isoniazid (50 $\mathrm{mg} \cdot \mathrm{kg}^{-1} /$ day) for 4 consecutive days and then treated as G2. Animals were sacrificed 12 hours after the last treatment, plasma was collected for TBARS analysis and the liver left lobe and both kidneys were removed for histological evaluation.

RESULTS: Results have shown increased TBARS levels in G3 and G4, with mild increase in G2. Histological evaluation has revealed focal liver necrosis in rats pretreated with isoniazid (G3).

CONCLUSION: Sevoflurane has promoted lipid peroxidation only when associated to isoniazid.

Key Words: ANESTHETICS, Volatile: sevoflurane; ANIMAL: rat; DRUGS: isoniazid; METABOLISM: lipid peroxidation

\section{INTRODUÇÃO}

A peroxidação de ácidos graxos insaturados nas memAbranas lipídicas é um processo conhecido como peroxidação lipídica. Este processo promove grave alteração da membrana celular, causando perda da fluidez, alteração da função secretora e dos gradientes iônicos transmembrana. Além disso, tem sido observada perda da seletividade na troca iônica, com liberação do conteúdo de organelas, levando à formação de produtos citotóxicos até a morte celu$\operatorname{lar}^{1}$. 
Trabalhos recentes têm correlacionado a toxicidade hepática e renal, provocada por anestésicos como o hidrocarboneto halogenado halotano ${ }^{2}$ e éteres como o isoflurano ${ }^{3}$, ao ataque aos ácidos graxos polinsaturados, presentes nas membranas plasmáticas, pelas espécies reativas do oxigênio produzidas durante a biotransformação desses agentes, indicando ser esse um dos possíveis mecanismos responsáveis pelas lesões teciduais.

$\mathrm{O}$ sevoflurano $\left(\mathrm{CH}_{2} \mathrm{~F}-\mathrm{O}-\mathrm{CH}\left(\mathrm{CF}_{3}\right)_{2}\right.$; fluorometil 2,2,2-trifluoro-1-[trifluorometil] etil éter) é um agente anestésico inalatório halogenado com flúor, apresentando baixa solubilidade sangüínea, o que justifica sua larga utilização em anestesia pediátrica e ambulatorial (indução e despertar rápidos) ${ }^{4,5}$. Sua biotransformação, catalisada principalmente pelo citocromo P450 2E1, é predominantemente hepática e pequena ( $2 \%$ a $5 \%$ ), com a produção do flúor inorgânico e do flúor orgânico: hexafluoroisopropanol (HFIP) ${ }^{6}$. Entretanto, o flúor inorgânico é capaz de produzir peroxidação lipídica em diversos tecidos como fígado, encéfalo e intestino de ratos ${ }^{7}$.

No fígado, monoxigenases, como a isoforma $\mathrm{P} 4502 \mathrm{E} 1$, possuem alta atividade de NADPH-oxidase através da qual espécies reativas do oxigênio são produzidas, como o ânion superóxido $\left(\mathrm{O}_{2}{ }^{-}{ }^{-}\right)$e o peróxido de hidrogênio $\left(\mathrm{H}_{2} \mathrm{O}_{2}\right)$ e, conseqüentemente, podem induzir à peroxidação lipídica potencialmente lesiva ${ }^{8}$.

Considerando-se que não há registros de estudos a respeito da lipoperoxidação após múltiplas exposições ao sevoflurano, o presente trabalho tem como objetivo avaliar a peroxidação lipídica em plasma de ratos Wistar tratados com sevoflurano e submetidos à indução enzimática do citocromo P450 2E1 com isoniazida.

\section{MÉTODO}

Após a aprovação pelo Comitê de Ética em Pesquisa Animal, 42 ratos machos Wistarpesando em média $280 \mathrm{~g}$ e com idade de 90 dias, compuseram os grupos do trabalho. Os animais receberam água e alimentos ad libitum e foram submetidos ao ciclo claro/escuro de 12 horas no biotério do Centro de Ciências da Saúde da Universidade Federal do Rio Grande do Norte (UFRN). Os ratos foram anestesiados em uma câmara de vidro de $3.200 \mathrm{~cm}^{3}$ usando-se fluxo de oxigênio a $100 \%$ de $1 \mathrm{I} \cdot \mathrm{min}^{-1}$ e um vaporizador calibrado HB44 (Abott, Madison, WI - EUA). Após 1 hora de exposição, variável de acordo com o grupo, os ratos receberam oxigênio a $100 \%$ com fluxo de $1 \mathrm{l} . \mathrm{min}^{-1}$ até recuperarem o reflexo postural, quando então foram colocados em suas gaiolas. Os animais foram distribuídos em quatro grupos: G1, grupo controle, que recebeu oxigênio a $100 \%$, com um fluxo de $1 \mathrm{I} \cdot \mathrm{min}^{-1}$ por $60 \mathrm{mi}-$ nutos, durante cinco dias consecutivos. O G2 recebeu sevoflurano a $4 \%(1,8 \mathrm{CAM} / \mathrm{h})$ em oxigênio a $100 \%$ com fluxo de 1 I. $\min ^{-1}$ por 60 minutos, durante cinco dias consecutivos. Os animais do G3 receberam injeção intraperitoneal de isoniazida, $\left(50 \mathrm{mg} \cdot \mathrm{kg}^{-1}\right)$ por dia, durante quatro dias consecutivos; em seguida, foram tratados com oxigênio a $100 \%$ sob fluxo de $11 . \mathrm{min}^{-1}$, por 60 minutos, durante cinco dias consecutivos. O G4 recebeu isoniazida ( $50 \mathrm{mg} \cdot \mathrm{kg}^{-1}$ ) por via intraperitoneal

Revista Brasileira de Anestesiologia

Vol. 54, № 5, Setembro - Outubro, 2004 durante quatro dias seguido do tratamento com sevoflurano a $4 \%$ em oxigênio a $100 \%$ sob fluxo de $11 . \mathrm{min}^{-1}$, por 60 minutos, durante cinco dias consecutivos. Após 12 horas da última exposição, os ratos foram sacrificados por deslocamento cervical e submetidos à laparotomia para coleta de sangue da veia porta e extração dos rins e do lobo esquerdo do fígado.

O sangue heparinizado foi centrifugado $1100 \times$ g a $4{ }^{\circ} \mathrm{C}$ por 10 minutos e o plasma separado foi utilizado para determinação das substâncias reativas ao ácido tiobarbitúrico (SRAT), por meio da determinação colorimétrica do produto da reação entre o ácido tiobarbitúrico e o malonildialdeído, produzido durante a quebra de lipídios peroxidados. Após a coleta do sangue, o plasma foi separado e submetido à reação com ácido tiobarbitúrico 0,86\% (TBA). Fez-se a leitura da absorbância a $535 \mathrm{~nm}$ e calculou-se a concentração de SRAT ${ }^{9}$, considerando-se um coeficiente de extinção molar $\varepsilon=0,156$ $\mu \mathrm{M}^{-1} . \mathrm{cm}^{-1}$. Os valores foram expressos em nmol. $\mathrm{I}^{-1}$.

Os espécimes de fígado e de rim foram fixados em formol a $10 \%$ e conduzidos ao laboratório de Anatomia Patológica da UFRN onde o mesmo patologista realizava a análise das lâminas e desconhecia os grupos a que pertenciam os espécimes. As amostras de tecido foram embebidas em parafina, cortadas com $4 \mu \mathrm{m}$ de espessura e coradas pela hematoxilina-eosina (HE) e pelo tricrômico de Masson. Os cortes foram então codificados e as modificações patológicas, examinadas através da microscopia ótica.

Acomparação entre todos os grupos foi realizada pela Análise de Variância seguida da aplicação do teste $U$ de Mann-Whitney para comparação entre dois grupos, com significância de $p \leq 0,05$.

\section{RESULADOS}

A concentração de SRAT no plasma dos ratos do grupo controle e dos ratos tratados com sevoflurano e submetidos ou não a pré-tratamento com isoniazida (INH) é mostrada na tabela I.

Tabela I - Determinação da Produção de Substâncias Reativas ao Ácido Tiobarbitúrico (SRAT) em Ratos Controle e Tratados com Sevoflurano Submetidos ou Não ao Pré-Tratamento com Isoniazida

\begin{tabular}{|c|c|c|}
\hline Grupos & Tratamento & $\mathrm{nmol} . .^{-1}$ \\
\hline G1 & Oxigênio & $2,08 \pm 0,17$ \\
\hline G2 & Sevoflurano & $2,37 \pm 0,09$ \\
\hline G3 & Isoniazida & $3,27 \pm 0,47$ \\
\hline G4 & Isoniazida + Sevoflurano & $3,41 \pm 0,53$ * \\
\hline
\end{tabular}

Os resultados estão expressos como Média \pm DP de 6 animais em cada grupo

${ }^{*}$ Indica diferença estatística em relação ao $\mathrm{G} 1(p \leq 0,055)$

Os ratos tratados com oxigênio e sevoflurano apresentaram elevação nas taxas de SRAT, embora não significativa quando comparada ao G1, tratado apenas com oxigênio. Já os animais tratados apenas com isoniazida - G3 apresentaram aumento na concentração de SRAT quando comparados ao 
grupo de animais tratados apenas com oxigênio - G1, contudo estatisticamente não significativo. Entretanto, os animais pré-tratados com isoniazida seguido do tratamento com sevoflurano - G4 apresentaram aumento significativo em relação ao $G 1$ ( $p \leq 0,055)$ e um aumento de aproximadamente $30 \%$ em relação ao G2. Estes resultados sugerem que, embora o sevoflurano cause elevação nas taxas de SRAT, apenas na presença da isoniazida foi observado um percentual significativo desse aumento indicando assim a presença ativa do processo de peroxidação lipídica.

Os cortes histológicos (Figuras 1 a 4) mostraram para o grupo de animais tratados com sevoflurano - G2, congestão em sinusóides e na veia hepática e nos animais pré-tratados com isoniazida - G3 necrose hepática focal e infiltrado linfocitário. Nos cortes de fígado dos animais que foram tratados com oxigênio - G1 e nos animais pré-tratados com isoniazida e tratados com sevoflurano - G4 não foram encontradas anormalidades histológicas.

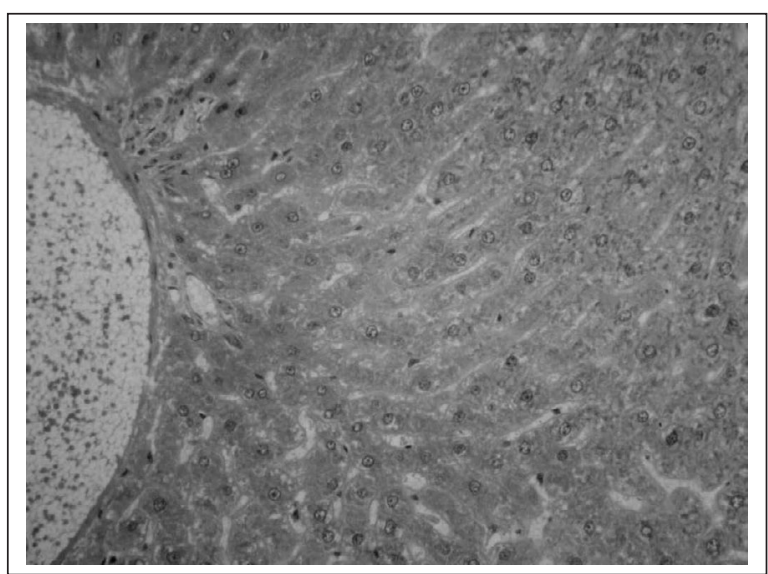

Figura 1 - Corte Histológico de Fígado Fixado com HE, obtido de Ratos Tratados com Oxigênio Aumento de 10x (G1)

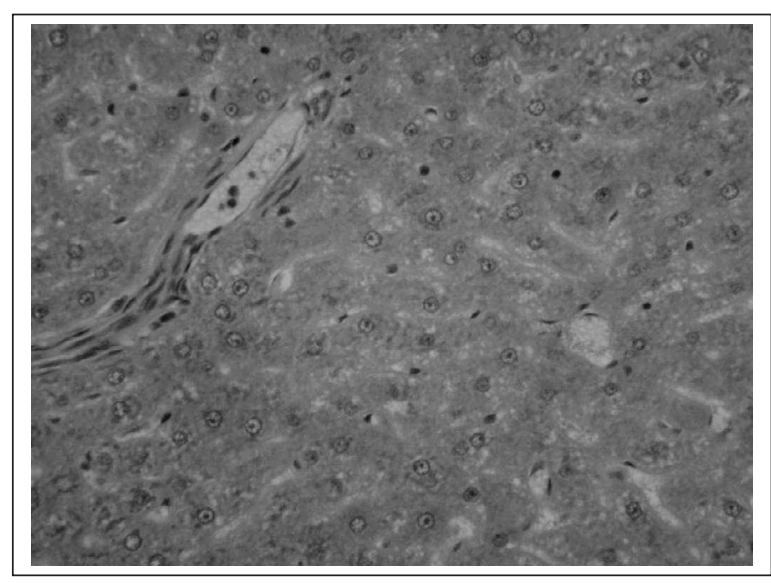

Figura 2 - Fotomicrografia de Corte Histológico de Fígado, obtido de Ratos Tratados com Sevoflurano. Coloração com HE

Aumento de 20x (G2)

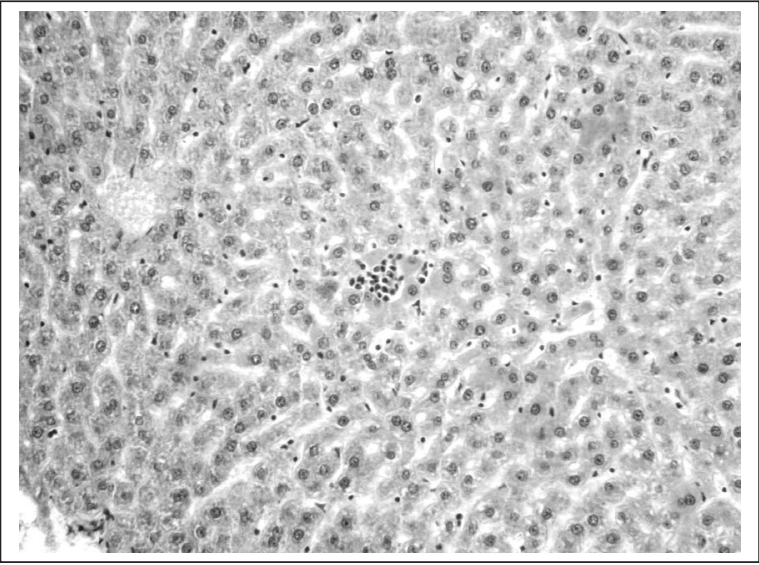

Figura 3 - Corte Histológico de Fígado Fixado com HE, obtido de Ratos Tratados com Isoniazida. Aumento 20x (G3) No centro da figura pode-se observar a necrose focal de um hepatócito com infiltrado linfocitário

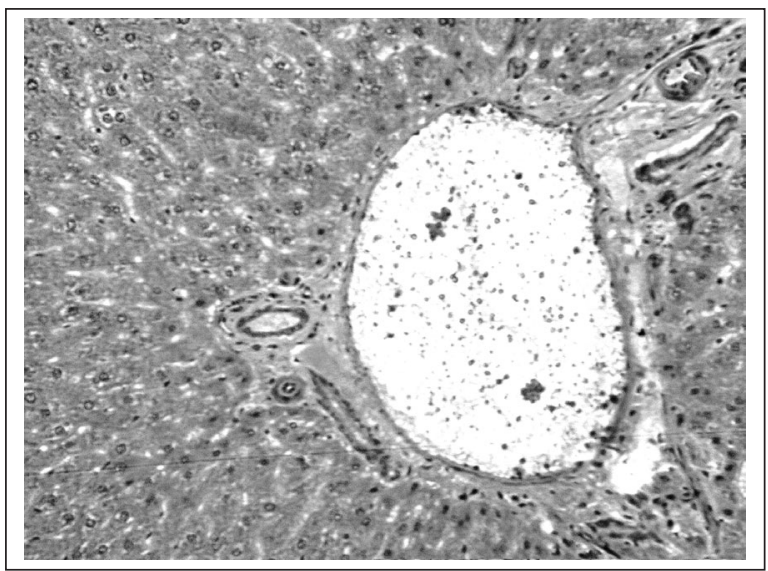

Figura 4 - Corte Histológico de Fígado Fixado com HE, obtido de Ratos Submetidos à Indução Enzimática com Isoniazida e Tratados com Sevoflurano. Aumento de 20x (G4)

O exame histológico dos cortes de rins (Figuras 5 a 8 ) dos grupos G2, G3 e G4 mostrou congestão glomerular. Nos animais tratados com oxigênio - G1 não houve anormalidades histológicas.

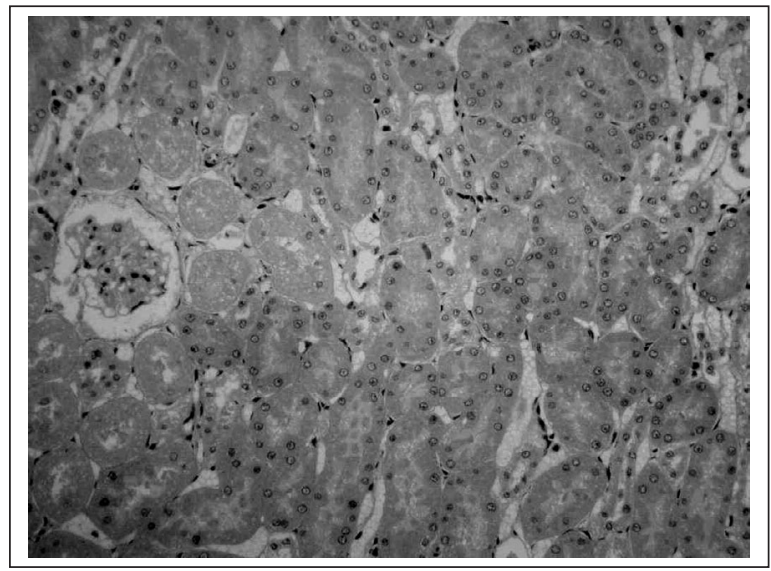

Figura 5 - Corte Histológico de Rim obtido de Ratos Tratados com Oxigênio, Grupo Controle. Corado com HE Aumento de 20x (G1)

Revista Brasileira de Anestesiologia Vol. 54, No 5, Setembro - Outubro, 2004 


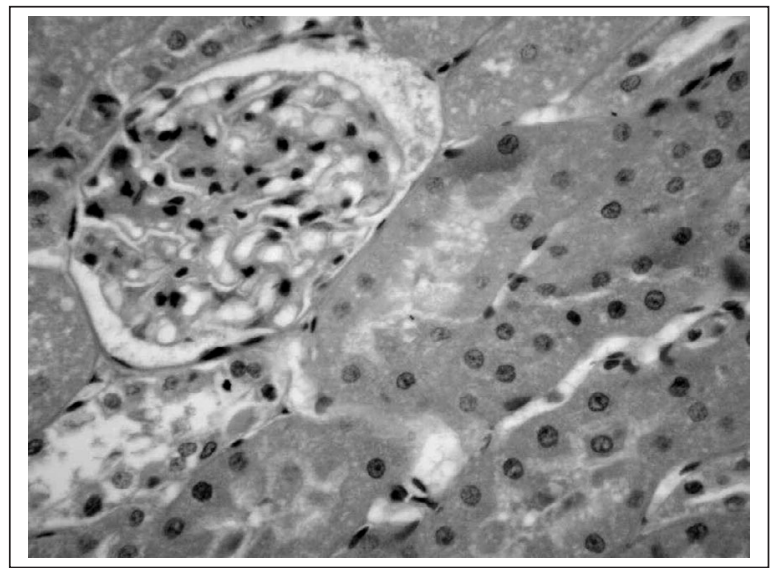

Figura 6 - Corte Histológico de Rim Fixado com HE, obtido de Ratos Tratados com Sevoflurano Aumento 40x (G2)

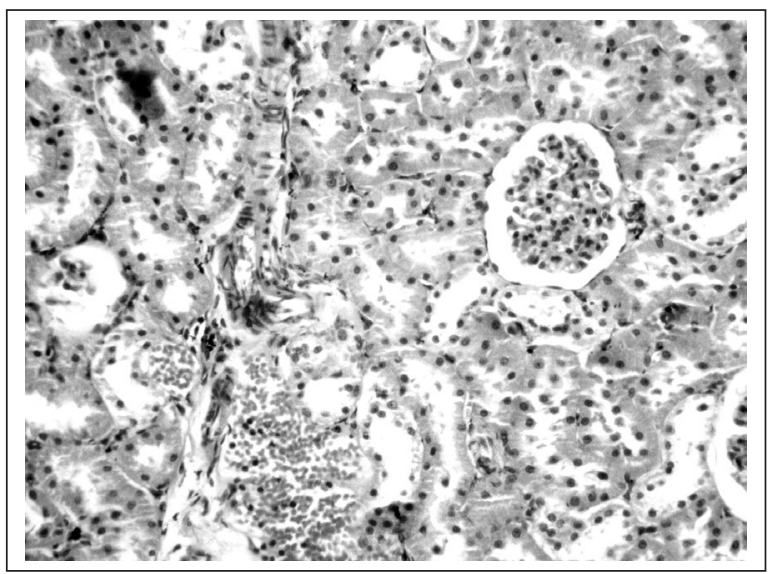

Figura 7 - Corte Histológico de Rim Fixado com HE, obtido de Ratos Pré-Tratados com Isoniazida Aumento 40x (G3)

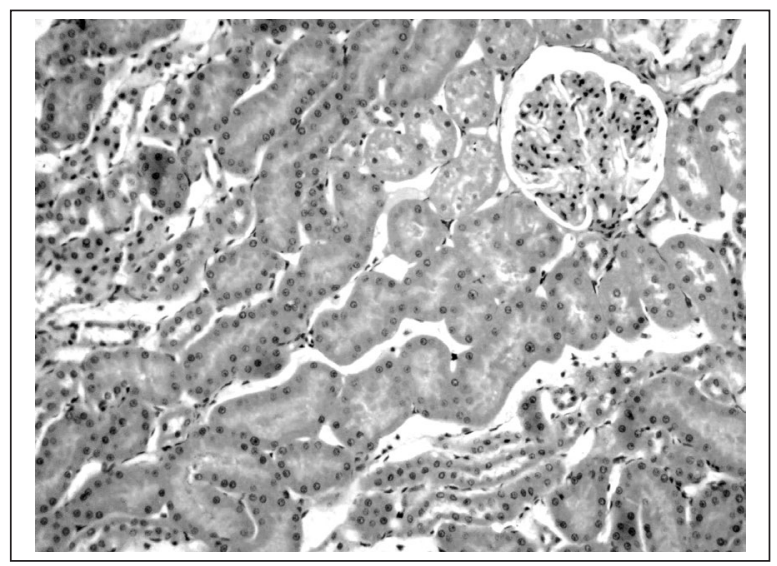

Figura 8 - Corte Histológico de Rim Fixado com HE, obtido de Ratos Submetidos à Indução Enzimática com Isoniazida e Tratados com Sevoflurano Aumento 20x (G4)

\section{DISCUSSÃO}

Durante os experimentos realizados, observou-se que o modelo de animais tratados com sevoflurano a $4 \%$ apresentou elevação dos níveis de SRAT, embora esse aumento não tenha sido estatisticamente significativo. Entretanto, esse aumento foi significativo na presença da isoniazida, um indutor enzimático do citocromo P450 2E1, o que permite sugerir ser o pré-tratamento com a isoniazida o responsável pelo aumento da peroxidação lipídica observada entre os diferentes grupos; o sevoflurano poderá também causar peroxidação lipídica de maneira significativa, apenas quando usado na presença da isoniazida (Tabela I).

O sevoflurano pode desencadear lipoperoxidação ${ }^{10}$, possivelmente através de seus metabólitos, como o flúor inorgânico, uma vez que este mostrou ser capaz de induzir a peroxidação lipídica em diversos tecidos, como fígado, encéfalo e intestino de $\operatorname{ratos}^{7}$, provavelmente por agir na compartimentalização do ferro ${ }^{11}$.

Também tem sido relatado que a isoforma P450 2E1, catalisadora da biotransformação do sevoflurano e reconhecida pela produção de espécies reativas do oxigênio, como o ânion superóxido $\left(\mathrm{O}_{2}{ }^{-}\right)$, e peróxido de hidrogênio $\left(\mathrm{H}_{2} \mathrm{O}_{2}\right)^{12}$, e o radical hidroxil $\left(\mathrm{OH}^{*}\right)$ é capaz de desencadear a peroxidação lipídica especialmente na presença de metais de transição ${ }^{8,13}$. Portanto, além da ação direta do flúor, a peroxidação lipídica observada nos ratos do grupo G4, pode ter sido causada pelo aumento na atividade do citocromo P450 2E1 induzida pela isoniazida.

Em contrapartida, Wang e col. ${ }^{14}$, utilizando coração isolado de ratos tratados com sevoflurano 1,5 CAM, submetidos à isquemia e reperfusão, encontraram diminuição dos níveis de peroxidação lipídica em relação ao grupo controle. Segundo Allaouchiche e col. ${ }^{15}$ o sevoflurano produz menos estresse oxidativo local e sistêmico em porcos quando comparado com o desflurano e sugere a possibilidade do mesmo ter alguma ação antioxidante.

Vale ressaltar que em relação ao hidrocarboneto halotano, existem estudos que tentam relacionar a sua toxicidade hepática à peroxidação lipídica ${ }^{16}$. Além dos agentes citados, o halogenado enflurano também tem sido relacionado como agente indutor da peroxidação lipídica ${ }^{17}$. Mais recentemente, estudos procuram demonstrar a possível atividade antioxidante de outros agentes anestésicos como o propofol, midazolam, cetamina e vecurônio ${ }^{18,19}$.

O fato de nenhum dos animais do grupo G4 ter apresentado necrose hepática pode estar relacionado à inibição enzimática na via de biotransformação da isoniazida, promovida pelo sevoflurano ou por algum de seus produtos de biotransformação como o flúor ou HFIP, como também ao fato de que este halogenado é capaz de manter a oferta de oxigênio aos tecidos, mesmo em situações em que o fluxo sangüíneo hepático possa estar diminuído ${ }^{20}$.

Aacetilisoniazida, o primeiro metabólito formado pela isoniazida, sofre hidrólise enzimática para formar ácido isonicotínico e acetilidrazina ${ }^{21}$. A acetilisoniazida e a acetilidrazina são capazes de produzir necrose hepática em ratos de forma 
dose-dependente ${ }^{22}$. O sevoflurano ou seus produtos de biotransformação poderiam estar inibindo a conversão da isoniazida à acetilisoniazida? Ou ainda, estariam eles contribuindo para o aumento na formação de ácido isonicotínico, que não é hepatotóxico, a partir da acetilisoniazida, ao invés de acetilidrazina? A verificação da concentração desses compostos nesse grupo de animais poderia ajudar a esclarecer estas hipóteses em estudos futuros. A isoniazida é um indutor enzimático específico do citocromo P450 2E1 capaz de aumentar, temporariamente, a toxicidade tecidual por meio do aumento na formação dos produtos derivados da biotransformação do fármaco utilizado. Teoricamente, ao aumentar a sua biotransformação aumenta a formação de seus bioprodutos realçando-lhes os efeitos adversos. No caso do sevoflurano, a isoniazida foi utilizada como indutor enzimático porque ela aumenta a taxa de desfluoração do sevoflurano de 0,5 a 4 vezes, de maneira dose e tempo-dependente ${ }^{23}$. Apenas nos animais do grupo G3 foi observada a presença de necrose focal e infiltrado inflamatório, indicando maior potencial hepatotóxico da isoniazida, ao contrário do grupo G4 onde o sevoflurano parece ter exercido um efeito protetor sobre a capacidade da isoniazida em produzir necrose hepática (Figura 3). Segundo a literatura a isoniazida é capaz de produzir necrose hepática de maneira dose/tempo dependente ${ }^{24,25}$.

Em relação à arquitetura renal, foi observada apenas congestão vascular presente em todos os grupos estudados. Não foram observados sinais patológicos clássicos de nefropatia por flúor, como picnose, cariorrexis, regeneração e dilatação dos túbulos renais ou mesmo hipereosinofilia, em nenhum dos grupos estudados após exposição aguda com lapso de tempo de $5 \mathrm{~h}$. Esse resultado está de acordo com a literatura que descreve exposição de ratos ao sevoflurano a $1,5 \%$ sem o uso de cal sodada durante 15 horas e nenhuma evidência histológica de lesão renal ${ }^{26}$.

Os resultados do presente trabalho contribuem com evidências de que o uso do sevoflurano em pacientes que fazem uso do tuberculostático isoniazida como medicamento ou em uso a longo prazo de álcool, ambos indutores do citocromo P450 2E1, pode levar à instalação do estresse oxidativo, uma vez que a defesa antioxidante não seria capaz de combater as substâncias pró-oxidantes geradas em maior proporção. Além disso, essa exposição, tornando-se prolongada ou repetitiva, poderia promover lesões eritrocitárias com conseqüentes complicações vasculares. Segundo Yesilkaya e col. ${ }^{27}$ alterações na capacidade de deformação eritrocitária podem resultar em problemas na perfusão tecidual e levar a complicações vasculares durante o período pós-anestésico. A peroxidaçãolipídica em eritrócitos também tem sido associada à diminuição da vida útil das hemácias e a estados hemolíticos ${ }^{28}$.

Deve, ainda ser considerado o uso do sevoflurano associado à isoniazida no caso de indivíduos portadores de deficiência da enzima glicose-6-fosfato desidrogenase (G6PD), que não conseguem ter produção satisfatória de NADPH, podendo se tornar mais susceptíveis a lesões peroxidativas do que indivíduos normais, sobretudo nas hemácias ${ }^{29}$. O NADPH é produzido no ciclo das pentoses pela G6PD e utilizado como doador do hidrogênio para restabelecer a atividade de enzimas antioxidantes presentes nos eritrócitos, como a glutationa e a catalase, que impedem o ataque das espécies reativas do oxigênio aos constituintes biológicos, como membranas plasmáticas, evitando lesões em sua estrutura.

Já é notória a maior segurança metabólica do sevoflurano no homem em relação ao rato, em função do maior teor de ß-liases presentes nos rins daqueles roedores ${ }^{30}$. Provavelmente, o uso isolado ou em anestesia balanceada, continua a ser seguro, pois a utilização do sevoflurano não induziu peroxidação lipídica significativa nos animais tratados apenas com esse agente anestésico.

\section{Thiobarbituric Acid Reactive Substances as an Index of Lipid Peroxidation in Sevoflurane-Treated Rats}

Francisco José Lucena Bezerra, M.D.; Adriana Augusto Rezende, M.D.; Sara Jane Rodrigues, M.D.; Maria das Graças Almeida, M.D.

\section{INTRODUCTION}

Unsaturated fatty acids peroxidation in lipid membranes is a process known as lipid peroxidation. This process promotes severe cell membrane changes resulting in loss of fluidity, changes in secreting function and transmembrane ionic gradients. In addition, the loss of ion selectivity has been observed with organelles content release, leading to cytotoxic product formation and even cell death ${ }^{1}$.

Recent studies have related liver and renal toxicity induced by anesthetic drugs such as halothane ${ }^{2}$ and ethers such as isoflurane ${ }^{3}$ to the attack of polyunsaturated fatty acids, present in plasma membranes, to oxygen reactive species produced during biotransformation of such agents, indicating that this could be one mechanism involved in tissue injuries. Sevoflurane $\left(\mathrm{CH}_{2} \mathrm{~F}-\mathrm{O}-\mathrm{CH}\left(\mathrm{CF}_{3}\right)_{2}\right.$; fluoromethyll 2,2,2-trifluoro -1-[trifluoromethyl] ethyl ether) is a fluorinated inhalational anesthetic agent, with low blood solubility, which justifies its widespread use in pediatric and outpatient anesthesia (fast induction and emergence $)^{4,5}$. Its biotransformation, especially catalyzed by cytochrome P4502E1, is low ( $2 \%$ to $5 \%$ ) and predominantly hepatic, with production of inorganic fluorine and organic fluorine: hexafluoroisopropanol (HFIP) ${ }^{6}$. However, inorganic fluorine is able to produce lipid peroxidation in different organs of rats, such as liver, brain and intestines ${ }^{7}$.

In the liver, monoxygenases, such as isoform P450 2E1, have high NADPH-oxidase activity through which oxygen reactive species are produced, such as superoxide anion $\left(\mathrm{O}_{2}{ }^{-}{ }^{-}\right)$and 
hydrogen peroxide $\left(\mathrm{H}_{2} \mathrm{O}_{2}\right)$ and, as a consequence, may induce potentially noxious lipid peroxidation ${ }^{8}$.

Considering that there are no reports on lipoperoxidation after multiple exposures to sevoflurane, this study aimed at evaluating lipid peroxidation in the plasma of Wistar rats treated with sevoflurane and submitted to cytochrome P450 $2 \mathrm{E} 1$ enzymatic induction with isoniazid.

\section{METHODS}

After the Animal Research Ethics Committee approval, 42 male Wistar rats weighing in mean $280 \mathrm{~g}$ and aged 90 days were involved in this study.

Animals received water and food ad libitum and were submitted to light/dark cycle of 12 hours in the experimental animals facility, Sciences Center, Federal University of Rio Grande do Norte (UFRN). Rats were anesthetized in a $3200 \mathrm{~cm}^{3}$ glass chamber with $1 \mathrm{~L}$. $\mathrm{min}^{-1}$ of $100 \%$ oxygen and a gauged HB44 (Abbott, Madison, WI-USA) vaporizer.

After 1-hour exposure, variable according to the group, rats received $1 \mathrm{~L}$. $\mathrm{min}^{-1}$ of $100 \%$ oxygen until postural reflex recovery, when they were placed in their cages.

Animals were distributed in 4 groups: G1, control group, received $1 \mathrm{~L}$. $\mathrm{min}^{-1}$ of $100 \%$ oxygen for 5 consecutive days; G2 received $4 \%$ sevoflurane $(1.8 \mathrm{MAC} / \mathrm{h})$ in $1 \mathrm{~L} . \mathrm{min}^{-1}$ of $100 \%$ oxygen for 5 consecutive days; $\mathrm{G} 3$ received intraperitoneal

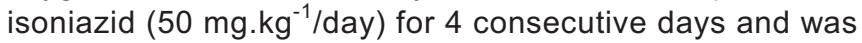
then treated with $1 \mathrm{~L}$. $\mathrm{min}^{-1}$ of $100 \%$ oxygen for 5 consecutive days; $\mathrm{G} 4$ received intraperitoneal isoniazid $\left(50 \mathrm{mg}^{\mathrm{kg}} \mathrm{kg}^{-1} /\right.$ day $)$ for 4 consecutive days and was then treated with $4 \%$ sevoflurane in 1 L. $\mathrm{min}^{-1}$ of $100 \%$ oxygen for 5 consecutive days. Rats were sacrificed 12 hours after the last exposure by cervical displacement and were submitted to laparotomy for portal vein blood collection and kidneys and liver left lobe removal.

Heparinized blood was centrifuged $1100 \times$ g at $4^{\circ} \mathrm{C}$ during 10 minutes and isolated plasma was used to determine thiobarbituric acid reactive substances (TBARS) through colorimetric determination of the product of the reaction between thiobarbituric acid and malonyldialdehyde produced during peroxidased lipid breakdown. After blood collection, plasma was separated and submitted to $0.86 \%$ thiobarbituric acid reaction. Absorbance was read at $535 \mathrm{~nm}$ and TBARS concentration was calculated ${ }^{9}$, considering a molar extinction coefficient $\varepsilon=0,156 \mu \mathrm{M}^{-1} \cdot \mathrm{cm}^{-1}$. Values were expressed in nmol. $L^{-1}$.

Liver and kidney specimens were fixed in $10 \%$ formalin and taken to the Pathologic Anatomy Lab, UFRN, where the same pathologist would analyze the specimens without knowing the group they belonged to. Tissue samples were soaked in paraffin, cut into $4 \mu \mathrm{m}$ slices and died with hematoxylin-eosin (HE) by Masson's trychromatic. Sections were then coded and pathologic changes were evaluated by optic microscopy. Analysis of Variance was used to compare among groups and Mann-Whitney $U$ test was used to compare between two groups, being significant $p \leq 0.05$.

\section{RESULTS}

Plasma TBARS concentration in control group rats and rats treated with sevoflurane and submitted or not to pretreatment with isoniazid (INH) is shown in table I.

Table I - Production of Thiobarbituric Acid (TBARS) Reactive Substances in Control Rats and Rats treated with Sevoflurane, submitted or not to Pretreatment with Isoniazid

\begin{tabular}{llc}
\hline Groups & Treatment & $\mathrm{nmol. \textrm {L } ^ { - 1 }}$ \\
\hline G1 & Oxygen & $2.08 \pm 0.17$ \\
G2 & Sevoflurane & $2.37 \pm 0.09$ \\
G3 & Isoniazid & $3.27 \pm 0.47$ \\
G4 & Isoniazid + Sevoflurane & $3.41 \pm 0.53 *$ \\
\hline
\end{tabular}

Results are expressed in Mean \pm SD for 6 animals in each group * Statistically significant as compared to G1 ( $p \leq 0.055)$

Rats treated with oxygen and sevoflurane presented high TBARS rates, although not significant as compared to $\mathrm{G} 1$ treated with oxygen alone. Conversely, animals treated with isoniazid alone - G3 - had increase in TBARS concentration as compared to animals treated with oxygen alone - G1. However, animals pretreated with isoniazid followed by sevoflurane - G4 - had a significant increase as compared to G1 ( $p \leq 0.055$ ) and approximately $30 \%$ increase as compared to $G 2$. These results suggest that, although sevoflurane increases TBARS rates, a significant percentage of this increase was observed in the presence of isoniazid, thus indicating the active presence of lipid peroxidation.

Cross-sections (Figures 1 to 4 ) have shown for animals treated with sevoflurane - G2 - congestion in sinusoidal and hepatic vein, and for animals pretreated with isoniazid - G3 focal liver necrosis and lymphocytic infiltrate. In liver cross-sections of animals treated with oxygen - G1 - and animals pretreated with isoniazid and treated with sevoflurane G4 - no histological abnormalities have been found.

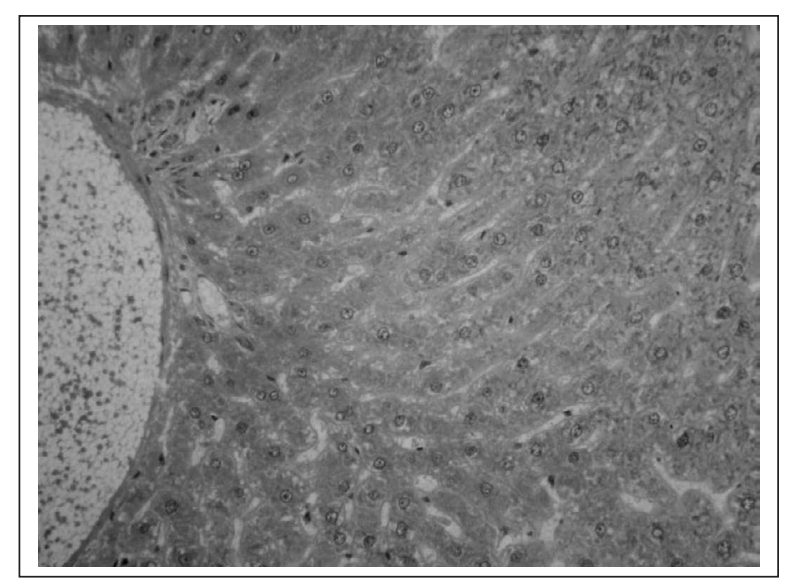

Figure 1 - Cross Section of the Liver fixed with HE, obtained from Rats Treated with Oxygen 10-fold Increase (G1) 


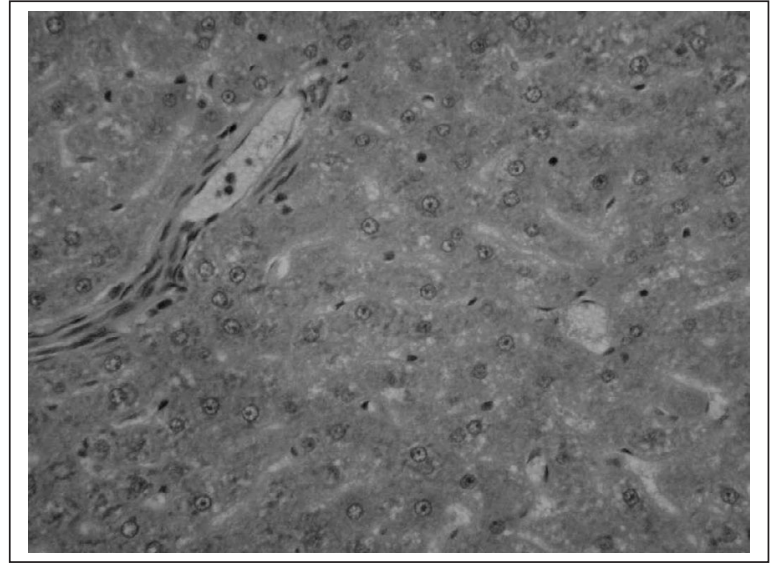

Figure 2 - Photomicrography of Liver Cross Section obtained from Rats Treated with Sevoflurane. Died with HE 20-fold Increase (G2)

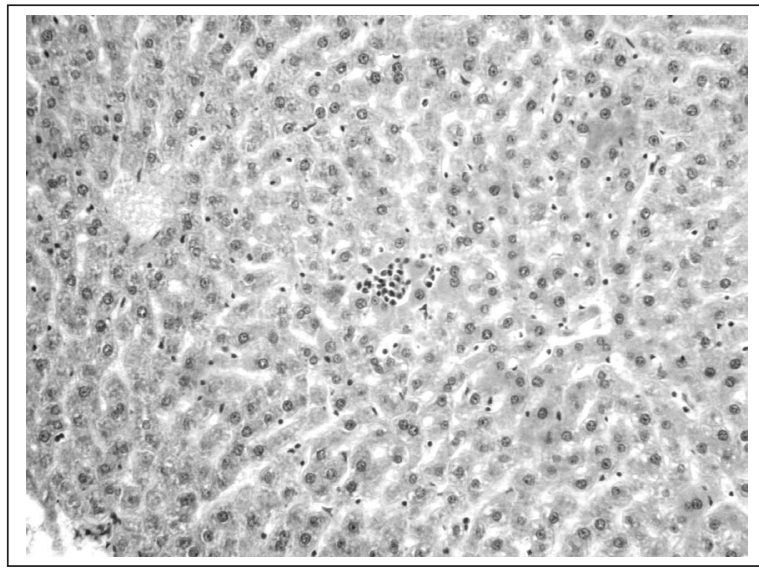

Figure 3 - Cross Section of Liver Fixed with HE, obtained from Rats Treated with Isoniazid 20 -fold Increase (G3)

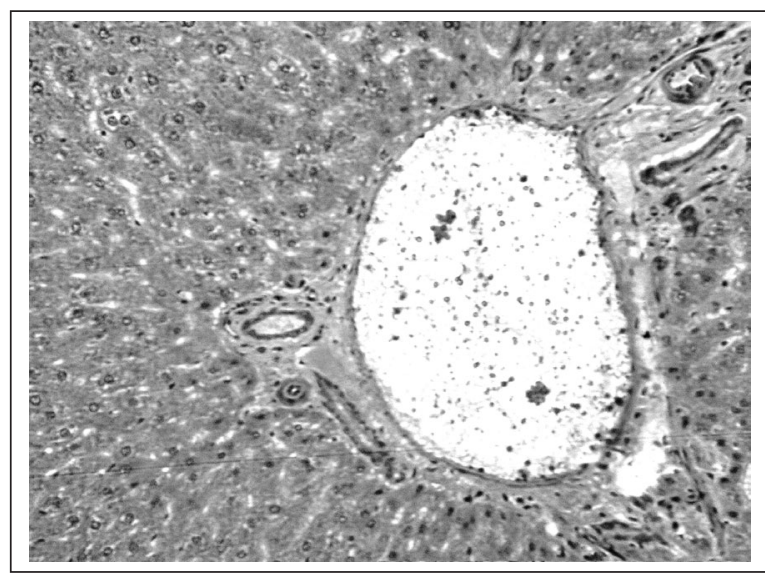

Figure 4 - Cross Section of Liver Fixed with HE, obtained from Rats Submitted to Enzymatic Induction with Isoniazid and Treated with Sevoflurane 20-fold Increase (G4)

In the center of the figure, focal necrosis of one hepatocyte with lymphocytic infiltrate may be observed.
Kidney histological evaluation (Figures 5 to 8 ) of groups G2, G3 and $\mathrm{G} 4$ has shown glomerular congestion. There have been no histological abnormalities in animals treated with oxygen-G1.

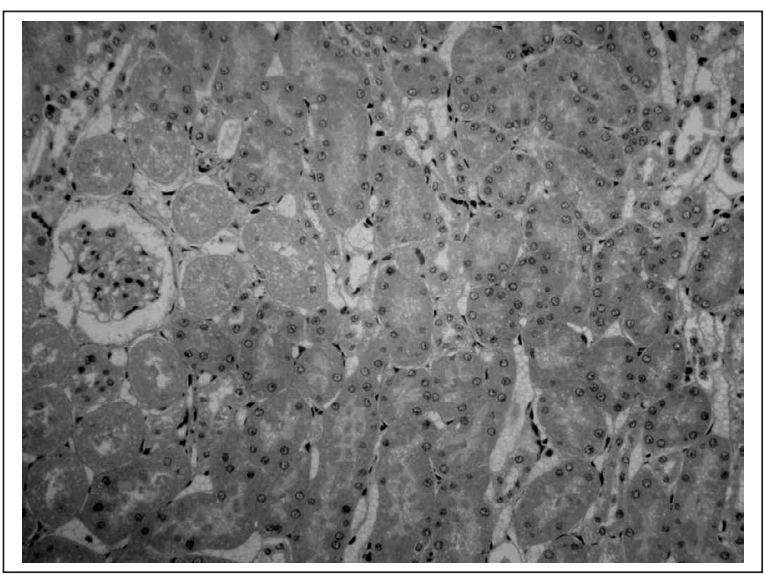

Figure 5 - Cross Section of Kidney obtained from Rats Treated with Oxygen, Control Group. Died with HE 20-fold Increase (G1)

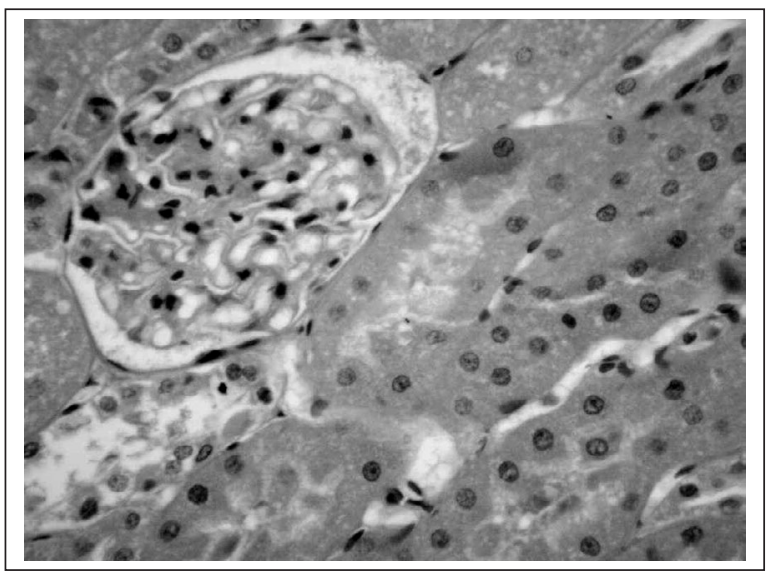

Figure 6 - Cross Section of Kidney Fixed with HE, obtained from Rats Treated with Sevoflurane 40-fold Increase (G2)

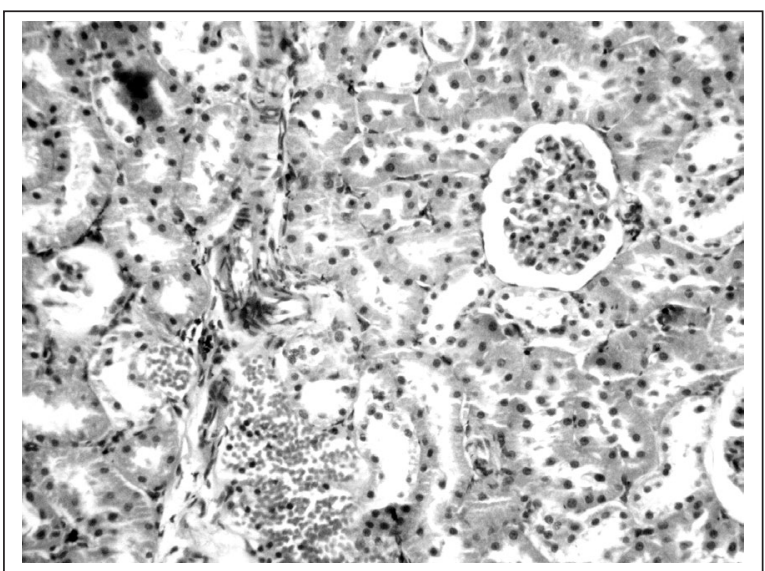

Figure 7 - Cross Section of Kidney Fixed with HE, obtained from Rats Pre-Treated with Isoniazid 40-fold Increase (G3)

Revista Brasileira de Anestesiologia Vol. 54, № 5, Setembro - Outubro, 2004 


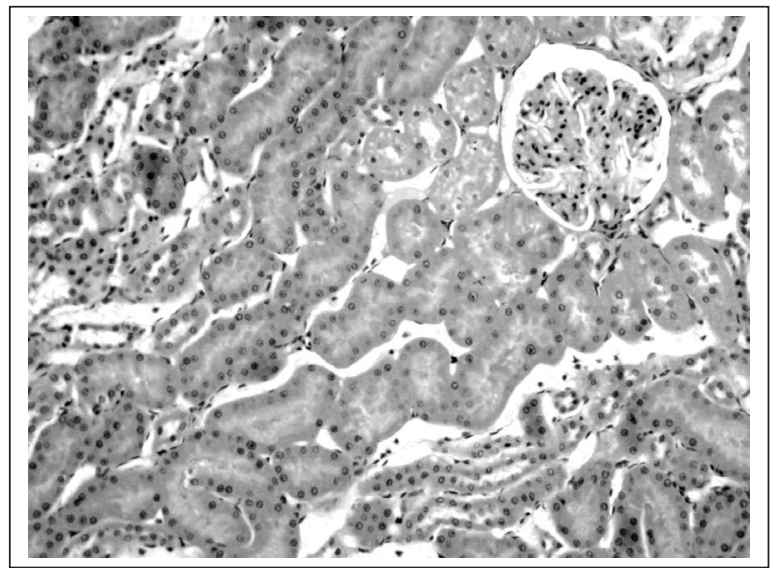

Figure 8 - Cross Section of Kidney Fixed with HE, obtained from Rats Submitted to Enzymatic Induction with Isoniazid and Treated with Sevoflurane 20-fold Increase (G4)

\section{DISCUSSION}

It has been observed during the experiments that animals treated with $4 \%$ sevoflurane have shown high TBARS levels, although without statistical significance. This increase, however, has been significant in the presence of isoniazid, an enzymatic inducer of cytochrome P450 2E1, allowing to suggest that pretreatment with isoniazid has been responsible for increased lipid peroxidation observed among different groups; sevoflurane may also induce significant lipid peroxidation, but only when in association with isoniazid (Table I).

Sevoflurane may trigger lipoperoxidation ${ }^{10}$, possibly through its metabolites, such as inorganic fluorine, since it was able to induce lipid peroxidation in different tissues, such as liver, brain and intestine of rats ${ }^{7}$, probably by acting on iron compartmentation ${ }^{11}$.

It has also been reported that isoform $\mathrm{P} 4502 \mathrm{E} 1$, sevoflurane biotransformation catalyst, is recognized by the production of oxygen reactive species, such as superoxide anion $\left(\mathrm{O}_{2}{ }^{{ }^{-}}\right)$ and hydrogen peroxide $\left(\mathrm{H}_{2} \mathrm{O}_{2}\right)^{12}$, in addition to the hydroxyl radical $\left(\mathrm{OH}^{\circ}\right)$ and the ability to trigger lipid peroxidation, especially in the presence of transition metals ${ }^{8,13}$. So, in addition to fluorine's direct action, lipid peroxidation observed in G4 rats, may have been caused by increased cytochrome P450 2E1 activity induced by isoniazid.

Conversely, Wang et al. ${ }^{14}$ using isolated hearts of rats treated with 1.5 MAC sevoflurane, submitted to ischemia and reperfusion, have found decreased lipid peroxidation levels in rats treated with sevoflurane as compared to control group. According to Allaouchiche et al. ${ }^{15}$ sevoflurane induces less local and systemic oxidative stress in swine as compared to desflurane, suggesting the presence of some antioxidant action.

It is worth highlighting that there are studies trying to relate halothane's liver toxicity to lipid peroxidation ${ }^{16}$. In addition to the above-mentioned agents, enflurane has also been reported as lipid peroxidation inducer ${ }^{17}$. More recent studies

Revista Brasileira de Anestesiologia

Vol. 54, No 5, Setembro - Outubro, 2004 have tried to show the potential antioxidant activity of other anesthetic agents such as propofol, midazolam, ketamine and vecuronium ${ }^{18,19}$.

The fact that no G4 animals had liver necrosis may be related to enzymatic inhibition in isoniazid biotransformation path, promoted by sevoflurane or some of its biotransformation products, such as fluorine or HFIP, as well as to the fact that this halogenate is able to maintain oxygen supply to tissues even in situations when liver blood flow may be decreased ${ }^{20}$. Acetylisoniazid, first metabolite formed by isoniazid, suffers enzymatic hydrolysis to form isonicotinic acid and acetylhydrazin ${ }^{21}$. Acetylisoniazid and acetylhydrazin are able to produce dose-dependent liver necrosis in rats ${ }^{22}$. Could sevoflurane or its biotransformation products be inhibiting isoniazid conversion into acetylisoniazid? Or even, would they be contributing for increased isonicotinic acid formation, which is not hepatotoxic, as from acetylisoniazid, as opposed to acetylhydrazin?

The evaluation of these compounds concentration in this group of animals could help explaining these hypotheses in further studies. Isoniazid is an enzymatic inducer specific of cytochrome P450 2E1 able to temporarily increase tissue toxicity by increasing the formation of products derived of the biotransformation of the drug used. In theory, increasing its biotransformation increases the formation of its bioproducts, worsening adverse effects. For sevoflurane, isoniazid was used as enzymatic inducer because it increases sevoflurane's defluorination rate in 0.5 to 4 times, in a dose and time dependent way ${ }^{23}$.

Focal necrosis and inflammatory infiltrate have only been observed in $\mathrm{G} 3$ animals, indicating higher isoniazid hepatotoxic potential, as opposed to G4 where sevoflurane seems to have had a protective effect over isoniazid's ability to produce liver necrosis (Figure 3). According to the literature, isoniazid is able to produce dose/time-dependent liver necrosis ${ }^{24,25}$. In terms of renal architecture, there has been vascular congestion in all studied groups. There have been no classic pathologic signs of fluorine-induced nephropathy, such as pyknosis, karyorrhexis, renal tubules regeneration and dilatation or even hypereosinophilia in all studied groups after acute exposure with a time period of 5 hours. This result is in line with the literature, which describes rats exposed to $1.5 \%$ sevoflurane without soda lime during 15 hours with no histological evidence of kidney injury ${ }^{26}$

Our results contribute with evidences that the use of sevoflurane in patients in treatment with isoniazid or in chronic use of alcohol, both cytochrome P450 2E1 inducers, may lead to oxidative stress, since the antioxidant defense would be unable to fight pro-oxidant substances generated in a higher proportion. In addition, this exposure, if prolonged or repeated, could promote erythrocytic injuries with consequent vascular complications. According to Yesilkaya et al. ${ }^{27}$ changes in erythrocytic deformation ability could result in tissue perfusion problems and lead to vascular complications in the postanesthetic period. Lipid peroxidation in erythrocytes has been also associated to decreased red blood cells useful life and hemolytic states ${ }^{28}$. 
Sevoflurane associated to isoniazid should also be considered for individuals with glucose-6-phosphate dehydrogenase enzyme (G6PD) deficiency, because they haven't satisfactory NADPH production and may be more susceptible to peroxidating injuries than normal individuals, especially in red blood cells ${ }^{29}$. NADPH is produced by G6PD during pentoses cycle and is used as hydrogen donor to biologic constituents, such as plasma membranes, preventing their structural damage.

The metabolic pathway of sevoflurane is more safe in men as compared to rats, due to the higher $\beta$-lyase content of those rodents' kidneys ${ }^{30}$. Possibly, its single use or during balanced anesthesia is still safe, because sevoflurane has not induced significant lipid peroxidation in the animals treated with it as unique agent.

\section{REFERÊNCIAS - REFERENCES}

01. Ferreira ALA, Matsubara SL - Radicais livres: conceitos, doenças relacionadas, sistema de defesa e estresse oxidativo. Rev Ass Med Brasil, 1997;43:61-68.

02. Durak I, Guven T, Birey M et al - Halothane hepatotoxicity and hepatic free radical metabolism in guinea pigs: the effects of vitamin E. Can J Anaesth, 1996;43:741-748.

03. Durak I, Ozturk HS, Dikmen B et al - Isoflurane impairs antioxidant defence system in guinea pig kidney. Can J Anaesth, 1999;46:797-802.

04. Delfino J, Vale NB, Magalhães E et al - Estudo comparativo entre sevoflurano e halotano para cirurgia pediátrica de curta duração. Rev Bras de Anestesiol, 1997;47:10-15.

05. Goa KL, Noble S, Spencer CM - Sevoflurane in paediatric anaesthesia: a review. Paediatr Drugs, 1999;1:127-153.

06. Kenna JG, Jones RM - The organ toxicity of inhaled anesthetics. Anesth Analg, 1995;81:(Suppl)S51-S66.

07. Shayiq RM, Raza H, Kidwai AM - Fluoride and lipid peroxidation: a comparative study in different rat tissues. Bull Environ Contam Toxicol, 1986;37:70-76.

08. Dai Y, Rashba-Step J, Cederbaum Al - Stable expression of human cytochrome P4502E1 in HepG2 cells: characterization of catalytic activities and production of reactive oxygen intermediates. Biochemistry, 1993;32:6928-6937.

09. Bernheim F, Bernheim MLC, Wilbur KM - The reaction between thiobarbituric acid and the oxidation products of certain lipids. J Biol Chem, 1948;174:257-264.

10. Sato N, Fujii K, Yuge O - In vivo and in vitro sevoflurane-induced lipid peroxidation in guinea-pig liver microsomes. Pharmacol Toxicol, 1994;75:366-370.

11. Kundu D, Hallinan T - Fluoride or GTP-gamma-S markedly stimulate lipid peroxidation catalysed by endogenous iron in rat liver microsomes. Biochem Soc Trans, 1995;23:541S.

12. Ekstrom G, Ingelman-Sundberg M - Rat liver microsomal NADPH-supported oxidase activity and lipid peroxidation dependent on ethanol-inducible cytochrome P-450 (P-450llE1). Biochem Pharmacol, 1989;38:1313-1319.

13. Arimoto T, Yoshikawa T, Takano $\mathrm{H}$ et al - Generation of reactive oxygen species and 8-hydroxy-2'-deoxyguanosine formation from diesel exhaust particle components in L1210 cells. Jpn J Pharmacol, 1999;80:49-54.
14. Wang J, Zeng YM, Li H - Effects of sevoflurane and halothane on contracture function, SOD activity, MDA content on isolated ischemic rat hearts. Anesthesiology, 1997;87:3A.

15. Allaouchiche B, Debon R, Goudable J et al - Oxidative stress status during exposure to propofol, sevoflurane and desflurane. Anesth Analg, 2001;93:981-985.

16. Wood CL, Gandolfi AJ, Van Dyke RA - Lipid biding of a halothane metabolite. Relationship to lipid peroxidation in vitro. Drug Metab Dispos, 1976;4:305-313.

17. Naziroglu M, Gunay C - The levels of some antioxidant vitamins, glutathione peroxidase and lipoperoxidase during anaesthesia of dogs. Cell Biochem Funct, 1999;17:207-212.

18. Tsuchiya M, Asada A, Kasahara E et al - Antioxidant protection of propofol and its recycling in erythrocyte membranes. Am J Respir Crit Care Med, 2002;165:54-60.

19. Kang MY, Tsuchiya M, Packer L et al - In vitro study on antioxidant potential of various drugs used in the perioperative period. Acta Anaesthesiol Scand, 1998;42:4-12.

20. Kharasch ED, Frink EJ, Artru A et al - Long-duration low-flow sevoflurane and isoflurane effects on postoperative renal and hepatic function. Anesth Analg, 2001;93:1511-1520.

21. Timbrell JA, Mitchell JR, Snodgrass WR et al - Isoniazid hepatotoxicity: the relationship between covalent binding and metabolism in vivo. J Pharmacol Exp Ther, 1980;213:364-369.

22. Mitchell JR, Zimmerman HJ, Ishak KG et al - Isoniazid liver injury: clinical spectrum, pathology, and probable pathogenesis. Ann Intern Med, 1976;84:181-192.

23. Rice SA, Sbordone L, Mazze RI - Metabolism by rat hepatic microsomes of fluorinated ether anesthetics following isoniazida administration. Anesthesiology, 1980;53:489-493.

24. Walubo A, Smith P, Folb PI - The role of oxygen free radicals in isoniazida-induced hepatotoxicity. Methods Find Exp Clin Pharmacol, 1998:20:649-655.

25. Sarich TC, Zhou T, Adams SP et al - A model of isoniazid-induced hepatotoxicity in rabbits. J Pharmacol Toxicol Methods, 1995;34:109-116.

26. Bermejo-Alvarez MA, Rodrigues-Dintén MJ, Brime-Casanueva $\mathrm{Jl}$ et al - Efectos renales de la anestesia prolongada com sevoflurane en ratas Wistar. Rev Esp Anestesiol Reanim, 1999;46:241-246.

27. Yesilkaya A, Ertug Z, Yegin A et al - Deformability and oxidant stress in the red blood cells under the influence of halothane and isoflurane anesthesia. Gen Pharmacol, 1998;31:33-36.

28. Saralakumari D, Rao PR - Erythrocyte glutathione metabolism in human chronic fluoride toxicity. Biochem Int, 1991;23:349-357.

29. Luzzatto L, Mehta A - The Metabolic Basis of Inherited Disease, $2^{\text {nd }}$ Ed, New York McGraw-Hill, 1995; 225-229.

30. Gentz BA, Malan TP - Renal toxicity with sevoflurane: a storm in a teacup? Drugs, 2001;61:2155-2162.

\section{RESUMEN}

Bezerra FJL, Rezende AA, Rodrigues SJ, Almeida MG - Determinación de las Substancias Reativas al Ácido Tiobarbitúrico como Indicador de la Peroxidación Lipídica en Ratones Tratados con Sevoflurano

JUSTIFICATIVA Y OBJETIVOS: El sevoflurano es un éter fluorado de baja solubilidad sanguínea y su biotransformación ocurre por medio del sistema enzimático hepático oxidativo que envuelve el citocromo P450 2E1. La peroxidación lipídica ocurre durante el proceso de biotransformación dos éteres sobre la acción del citocromo P450, uno de los posibles mecanismos de toxicidad hepática y renal promovida por eses 
compuestos. El objetivo de este estudio fue determinar los niveles de substancias reactivas al ácido tiobarbitúrico (SRAT), como indicador de la peroxidación lipídica, en ratones que recibieron sevoflurano, previamente tratados o no con isoniazida, inductora enzimática del citocromo P450 2E1.

MÉTODO: Los animales fueron distribuidos aleatoriamente en 4 grupos que recibieron respectivamente: G1 - oxígeno a 100\% 1 l. $\mathrm{min}^{-1} / 60$ minutos por 5 días consecutivos; G2 - sevoflurano a $4 \%$ en oxígeno a $100 \%, 1$ l. $\mathrm{min}^{-1} / 60$ minutos por 5 días consecutivos; G3 - isoniazida (50 $\mathrm{mg}^{\mathrm{kg}}{ }^{-1}$.dia) por vía intraperitoneal durante 4 dias consecutivos, en seguida fue tratado como el G1, en $\mathrm{G} 4$ - isoniazida $50 \mathrm{mg}^{\mathrm{kg}}{ }^{-1}$. dia por vía intraperitoneal durante 4 días consecutivos, siendo tratado, posteriormente, como el G2. Después de 12 horas del último tratamiento, se sacrificaran los animales y fue colectado el plasma para la análisis de las SRAT, siendo removido el lóbulo izquierdo del hígado y de los riñones para examen histológico.

RESULTADOS: Los resultados mostraron aumento en las tasas de SRAT en el G3 y G4, con elevación discreta en G2. El estudio histológico reveló necrosis focal en el hígado de ratones pre-tratados con isoniazida (G3).

CONCLUSIONES: EI sevoflurano promovió peroxidación lipídica apenas cuando asociado a la isoniazida. 